\title{
as ciudades latinoamericanas en la economía mundial: la geografía de centralidad económica y sus transformaciones recientes
}

Profesor de Geografia Económica, Universidad de Hamburgo, Alemania. Profesor visitante de la Facultad de Economía, UNAM christof.parnreiter@uni-hamburg.de

\author{
Christof Parnreiter ${ }^{1}$
}

Journal of Economic Literature (JEL): $\mathrm{R} 1, \mathrm{O} 18, \mathrm{O} 57$

Palabras clave:

Economía espacial general

Análisis regional, urbano y rural

Estudios comparativos de países

Keywords:

General Spatial Economics Regional, Urban and Rural Analysis Comparative Studies of Countries

\section{Resumen}

En este artículo se analiza la posición de América Latina en la economía mundial y como ha cambiado en el transcurso del crisis de 2008/2009. Yendo más allá de los rankings habituales del PIB, se hará énfasis en la geografía de la centralidad económica. Se conceptualiza centros económicos como ciudades que concentran las actividades económicas relativamente monopolizadas. Una manifestación particular de core-ness económico son las ciudades globales. En el artículo se analizan datos sobre la localización de las empresas más grandes del mundo (Forbes Global 2000), el valor añadido bruto en los servicios al productor en 126 ciudades en todo el mundo, y la 'Global Network Connectivity' (GNC) de las ciudades globales calculado por GaWC (Globalization and World City Study Group). Los resultados muestran, primero, que en cuanto al poder económico formal, las ciudades latinoamericanas quedan marginales, aunque lograron mejorar su posición en el curso de la reciente crisis de la economía mundial. Segundo, los datos también revelan que São Paulo y la Ciudad de México contienen clusteres importantes de servicios al productor lo que las hace nodos importantes para la organización y la gestión de cadenas productivas globales. Sin embargo, para una discusión de la posición de América Latina en la centralidad de la economía mundial es clave comprender que los actores del poder organizativo y globalizador de sus ciudades en muchos casos son oficinas locales de empresas globales. Por eso se puede concluir que la centralidad que algunas ciudades latinoamericanas tienen en la economía mundial resulta de sus relaciones exógenas más que de su potencia endógena.

\section{Abstract}

In this paper I analyze the position of Latin America in the world economy and how it has change during the crisis of 2008/9. Going beyond the usual rankings of the GDP, the paper emphasizes the geography of economic centrality. Econo- 
mic centers are conceptualized as cities which concentrate economic activities which are relatively monopolized. One particular manifestation of this core-ness are the global cities. In this paper data on the localization of the world's biggest companies (Forbes Global 2000) are as well analyzed as data on gross value added in producer services in 126 cities around the world and the Global Network Connectivity (GNC) of the global cities, as calculated by GaWC (Globalization and World City Study Group). The results show, firstly, that as regards formal economic power Latin American cities remain marginal, though they succeeded to improve their position during the recent economic crisis. Secondly, data also show that São Paulo and Mexico City have important clusters of producer services, what makes them important nodes in the organization and governance of commodity chains. However, for a discussion of the position of Latin America in the economic centrality of the world economy it is crucial to understand that the agents in this organization and governance of commodity chains are in many cases local offices of global firms. This leads to the conclusion that the centrality that some Latin American cities have in the world economy results from their exogenous relations rather then from their endogenous economic potential.

\section{Introducción}

Mientras China se convirtió en la economía más grande del mundo en la segunda mitad de 2014 (IMF 2014a), el diagnóstico de las organizaciones internacionales para las economías latinoamericanas es más reservado. Se comprueba, por un lado, un crecimiento considerable en la última década y una reducción notable de la pobreza (Ferreira, et. al. 2013), pero también se reconocen serios desafíos. La OCDE $(2013,15)$, por ejemplo, espera un "deterioro de las perspectivas de crecimiento", lo cual "acentúa los retos estructurales que enfrenta América Latina", mientras el FMI (IMF 2014b, 14) concluye que "the balance of risks to the outlook is still tilted to the downside", básicamente por una demanda interna débil, la caída en los precios de materias primas y un empeoramiento en las condiciones financieras.

Ante esta situación, este artículo analiza la posición de América Latina en la economía mundial y como ha cambiado en el transcurso del crisis de 2008-2009. El propósito es, sin embargo, ir más allá de los rankings habituales del PIB o del PIB per cápita. Se hará particular énfasis en la geografía de la centralidad económica, es decir, en cuestiones de core-ness económico en vez del puro tamaño.

Esta perspectiva se desarrolla teóricamente en las dos primeras secciones de este artículo. Basado tanto en los análisis del sistema mundial como en la literaturas sobre cadenas productivas y ciudades globales, se resalta, primero, que los centros económicos surgen a raíz de la concentración de actividades económicas relativamente monopolizadas, $y$, segundo, que la regionalización de esta centralidad económica tipicamente son ciudades, y, en particular, las llamadas

\footnotetext{
1 El autor agradece el apoyo económico del DAAD para realizar una estancia como profesor invitado en la Facultad de Economía de la UNAM, México.
} 
ciudades globales. A partir de estas premisas, en la tercera sección se analizarán datos aptos para identificar la posición de América Latina en la geografía de la centralidad económica: la localización de las empresas más grandes del mundo (Forbes Global 2000), el valor bruto añadido en los servicios al productor en 126 ciudades en todo el mundo, y la Global Network Connectivity (GNC) de las ciudades globales calculado por GaWC. En la cuarte sección se discute y interpreta los resultados empíricos.

\section{La centralidad económica}

El punto de partida de esta reflexión sobre la centralidad en la economía mundial es la noción de que el capitalismo se ha desarrollado a través de y se sostiene en divisiones de trabajo funcionales que toman la forma de cadenas productivas globales (Gereffi/Korzeniewicz 1994). Cabe subrayar que en estas divisiones del trabajo existe una interrelación entre la organización social y espacial de la economía (Wallerstein, 1983): los procesos de producción están repartidos en segmentos, con cada segmento tiene asignado tanto un valor específico (expresado en la parte del valor agregado total que esta secuencia logra aglutinar) como un lugar particular para ser desarrollado.

En cuanto a la organización social de la economía hay que subrayar que los procesos laborales que logran concentrar una buena parte del valor agregado son altamente renumerados porque consisten en actividades relativamente escasas y de gran demanda. Así, sus productores gozan de un monopolio relativo, ya que sus productos son difíciles de reemplazar (p.e. porque son intensivos en capital y en conocimientos o porque están protegidos por altas barreras de entrada). Controlando un proceso laboral relativamente monopolizado establece una posición central en cadenas productivas, dotada con poder superior en la gestión económica:

(T)he relative power of actors within a network depends, in large part, on the extent to which each possesses assets sought by the other party and the extent to which access to such assets can be controlled. The scarcer the asset the greater the bargaining power it conveys and vice versa (Coe et. al. 2008, 276).

Segundo, y en cuanto a la organización espacial de la producción, cabe señalar que los productores controlando procesos laborales relativamente monopolizados geográficamente no se distribuyen de manera igual. Más bien, a lo largo de la historia algunas regiones lograron concentrar de manera desproporcional los procesos productivos altamente renumerados, mientras otras regiones están caracterizadas por una mayoría de procesos laborales poco renumerados. A estas se les llama periferias 'zones that lost out in the distribution of surplus' (Wallerstein, 2001, 109). Los centros, por el contrario, se distinguen por un predominio de los procesos de alto rendimiento. Ya que estas actividades son, como se señalaba, relativamente escasas, en términos geográficos se debe conceptualizar la cen- 
tralidad o un centro como un nodo en varias cadenas productivas. Como dicen Hopkins y Wallerstein $(1977,114)$ :

(S)ome areas appear as cores or centers, in virtue of the many relational sequences leading from or to them'.

Poco después, Wallerstein $(1983,30)$ ya aplicó el término 'cadena productiva' apuntando que:

'commodity chains have not been random in their geographical directions. Were they all plotted on maps, we would notice that they have been centripetal in form. Their points of origin have been manifold, but their points of destination have tended to converge in a few areas. That is to say, they have tended to move from the peripheries of the capitalist world-economy to the centres or cores.

A pesar de que estas nociones conllevan una connotación muy espacial, Wallerstein nunca desarrolló una conceptualización geográfica exhaustiva de le centralidad en cadenas productivas. Ni lo hicieron otros autores en este campo de investigación, por lo cual muchos de los análisis de cadenas productivas carecen de un tratamiento geográfico profundo (Leslie y Reimer, 1999). Aún menos interés ha mostrado Wallerstein en integrar una perspectiva urbana en su reflexión -la ciudad como unidad de investigación queda practicamente ausente de la mayor parte de los análisis del sistema mundial- y, sobre todo, de las aportaciones teóricas (para un resumen de los pocos trabajos al respecto ver Smith, 2000). Sin embargo, existen buenas razones para conzeptualizar la centralidad económica en términos urbanos, lo que haré en la próxima sección.

\section{Ciudades como centros económicos}

La literatura -ya muy extensa- sobre ciudades globales apunta que estas operan como 'highly concentrated command points in the organization of the world economy' (Sassen, 1991, 3). Según Sassen, las ciudades globales obtienen su centralidad en la economía mundial por ser lugares donde se producen y se prestan los servicios al productor, considerados como indispensables para las operaciones tanto de empresas globales como para los mercados financieros (UNCTAD 2004; Bryson y Daniels, 2007). Gracias a sus clústeres de servicios al productor, las ciudades globales son nodos esenciales para el funcionamiento y la gestión de cadenas productivas (Brown, et al. 2010; Parnreiter, 2010) por lo cual es indispensable considerarles en un análisis de centralidad económica.

Para una conceptualización más general del papel de las ciudades en la economía cabe recordar que "we can say that the most basic raison d'être for cities... resides in their role as centers of economic production and exchange" (Scott/ Storper, 2014, 6). Hoy en día, 80\% del PIB global se produce en ciudades, con tan sólo 600 ciudades sumando $65 \%$ del crecimiento de la economía mundial 
(World Bank, 2013; MGI, 2012). Más aún, las ciudades son la regionalización típica de la centralidad económica porque abarcan y engendran dos prerrequisitos elementales para la expansión económica. Primero, en ellas se concentra la infraestructura física necesitada para la acumulación del capial, ya que ésta demanda grandes inversiones, lo cual las hace sensitivas a economías de escala. El resultado es su concentración geográfica, con las ciudades resultado de esta concentración:

It is impossible to imagine such a material process without the production of some kind of urbanization as a 'rationale landscape' within which the accumulation of capital can proceed. Capital accumulation and the production of urbanization go hand in hand" (Harvey, 1985, 190).

Gracias a la concentración espacial del capital fijo, cada ciudad es un:

"specific resource complex of humanly created assets to support both production (fixed capital) and consumption (the consumption fund)".

Funciona, por ende, como palanca para el desarrollo económico, ya que:

" $(\mathrm{t})$ he stock of fixed capital and of consumption fund assets does, however, provide a solid form of wealth that can be used to produce and consume more wealth (ibid., 144, mi énfasis).

La segunda razón para que las ciudades sean la regionalización típica de la centralidad económica es porque engendran y atribuyen lo que Schumpeter (1987 [1934], 100; mi traducción) llama 'nuevas combinaciones' y lo que Jacobs (p. e. 1970) distingue como 'nuevo trabajo': "(A)t the core of all processes of city growth is this root process ... (of) adding new kinds of work to other kinds of older work" (Jacobs 1970, 50f). La capacidad de producir innovación es, según Jacobs, algo exclusivamente urbana, resultando de las condiciones específicas de ciudades y, en particular, de los desafios que emergen de la aglomeración: Enfrentados diariamente con diversidad y densidad, con lo nuevo, extraño e inesperado, los ciudadanos a la fuerza tienen que ser inovadores para resolver los muchos problemas resultado de esta dinámica. Jacobs infiere, entones, que:

(c)ities are the mothers of economic development, not because people are smarter in cities, but because of the conditions of density. There is a concentration of need in cities, and a greater incentive to address problems in ways that haven't been addressed before. This is the essence of economic development (Jacobs, 1997).

\section{Aspectos metodológicos}

La tarea de operacionalizar estos razonamientos para un análisis empírico de la geografía de centralidad implica, primero, identificar las actividades económicas que aglutinan la mayor parte del valor agregado en una cadena productiva. En 
general, son las actividades en las cuales el margen entre los precios de los insumos y el precio final es más grande y que por ende generan las ganancias y los salarios más altos (p. e. industria manufacturera de alta tecnología o servicios intensivos en conocimientos). Segundo, se requieren datos sobre estas actividades económicas centrales, tanto a nivel global (para hacer una comparación geográfica exhaustiva) como, según el razonamiento de arriba, a nivel de ciudades. Sin embargo, son muy pocas las bases de datos a las que se puede recurrir con estas exigencias, ya que las instituciones internacionales, que ofrecen datos para una comparación global, suelen proporcionarlos por naciones. Tercero, y dado el interés en examinar la geografía de centralidad económica y la posición de América Latina en ella, hay que enfocarse en las actividades económicas centrales más relacionadas con la gestión de cadenas productivas.

Ante estas exigencias, aquí se examinan los siguientes datos. Primero, el análisis del patrón espacial de las sedes de las empresas más grandes del mundo (Forbes Global 2000²) informará sobre la geografía del poder formal en cadenas productivas. Los corporativos son, como lead firms de una multitud de cadenas productivas, actores centrales en la economía mundial (p.e. UNCTAD 2013), y sus casas matrices son los centros de la gestión de estas cadenas: "The corporate headquarters is the locus of overall control of the entire TNC" (Dicken, 2011, 134). Ahora, como Dicken mismo y otras investigaciones sobre la estructura y el funcionamiento de las corporaciones reconocen, la capacidad de la administración central en la sede de una empresa de gestionar y controlar la cadena productiva está incrustada y depende de:

a mosaic of relationships between its headquarters and subsidiaries forming an integrated network across borders. In addition to its internal network, the MNE (la empresa multinacional, C. P.) is part of a larger network external to its ownership boundaries, which may involve close coordination with other firms in supplier or customer relationships, or with strategic alliance partners (Dunning y Lundan, 2008, 117).

Como se ha indicado ya, entre las empresas externas que juegan un papel clave en las cadenas productivas sobresalen las de servicios al productor. Interesado no tanto en el poder económico formal -representado por la sede de una empresa- sino en 'the practice of global control: the work of producing and reproducing the organization and management of a global production system and a global marketplace for finance' (Sassen, 1991, 6), Sassen propone enfocarse en los proveedores de servicios al productor como actores centrales de la gestión económica. Siguiendo este razonamiento, el segundo indicador cuyo patrón geográfico se analizará aquí es el valor agregado bruto en servicios al productor, ${ }^{3}$ que informará

2 Forbes Global 2000 alista las 2000 empresas más grandes del mundo cotizadas en bolsas de valores. Aunque eso implique la desventaja de no contener empresas estatales o en propiedad familiar, se dio preferencia a la lista de Forbes hacia el listado semejante de Fortune Global 500 por la cobertura numérica mucho más amplia.

3 La fuente empírica que se utiliza es Passport Cities de Euromonitor International Ltd., una empresa 
sobre la geografía del poder organizativo en cadenas productivas detrás del poder formal de la casa matriz.

Dado el enfoque de este artículo en la centralidad en la economía mundial se necesita, sin embargo, concretizar la operacionalización aún más. Cabe mencionar que en cuanto a la capacidad de organizar y controlar cadenas productivas globales, dentro de las empresas de servicios al productor destacan las que operan a nivel mundial (para un resumen de las más importantes véase Taylor et. al. 2011). Son ellas -y solamente ellas- que pueden servir a sus clientes en donde sea con la misma cualidad, ya que combinan la ventaja de cercanía al cliente (lo local) con acceso a recursos y conocimiento implícito en lugares y ambientes desconocidos (lo global). Por ende, el poder organizativo a nivel mundial de una empresa de servicios al productor depende críticamente de su integración en una red global. Para medir este poder globalizador se analizará aquí, como tercer indicador, el patrón geográfico de la 'Global Network Connectivity' (GNC) de las ciudades globales. Calculado por GaWC (www.lboro.ac.uk/gawc/), la GNC estima las conexiones interurbanas -flujos de información, capital, profesionales, etc.- con base en un análisis del sector de los servicios al productor que cada ciudad global tiene (para la metodología ver Taylor, 2004). ${ }^{4}$

\section{Resultados empíricos}

En cuanto al primer indicador que sirve para evaluar el poder formal en cadenas productivas destacan Tokio, Beijing, Londres, Paris y Nueva York como ciudades cuyas empresas tienen más participación en el total de las ventas de las Forbes Global 2000. En cuanto a las ganancias, es Beijing la ciudad con la mayor participación, seguida por Nueva York, Tokio, Londres y Washington (para resultados más detallados ver cuadro 1). ${ }^{5}$ Las ciudades latinoamericanas aparecen solo marginalmente en el mapa de los corporativos más grandes del mundo: sus empresas generan en su conjunto $2.5 \%$ tanto de las ventas como de las ganancias de las Forbes Global 2000, lo que equivale aproximadamente a los valores de Seúl ( $2.6 \%$ de las ventas) y a Moscú ( $2.7 \%$ de las ganancias). De las 250 ciudades con más participación, solamente nueve son de América Latina. Referente a las ventas, São Paulo y Rio de Janeiro, ocupando los lugares 34 y 35, son las ciudades latinoamericanas mejor clasificadas, junto a Cincinnati. La Ciudad de México viene en el lugar 65, comparable con ciudades como Montreal o Viena, y Santiago de Chile sigue en el lugar 93. En cuanto a la participación en las ganancias generadas por las Forbes Global 2000, es la Ciudad de México la de más participación, colocándose en el lugar 41, detrás

privada de estudios de mercado. Passport Cities contiene datos sobre el valor agregado bruto en servicios financieros, inmobiliarios e actividades de negocio para 126 ciudades en todo el mundo.

4 La fuente empírica que se utiliza es la base de datos de GaWC (www.lboro.ac.uk/gawc/). Para realizar su analisis de red, GaWC analiza 200 empresas de servicios al productor con oficinas en 525 ciudades.

5 Agradezco a Csomós György por el apoyo en el análisis de los datos. 
de Cincinnati, seguida por São Paulo en el lugar 43 (comparable con Bangkok), Bogotá (67) y Rio de Janeiro (68).

Si bien es cierto que las ciudades latinoamericanas figuran solamente en los márgenes de los mapas de las ventas y ganancias de las empresas más grandes del mundo, cabe también señalar que la crisis de los años 2008/2009 tuvo un impacto menos fuerte en esta región que el promedio. Entre 2006 y 2014 las empresas con sede en América Latina incrementaron sus ventas 3.4 veces más rápidas que el promedio de las Forbes Global 2000 y las ganancias 55\% más rápidos. Destacan, en cuanto al incremento absoluto de las ventas, las ciudades brasileras, con São Paulo y Rio de Janeiro colocándose entre las 25 ciudades con las empresas más expansivas (cuadro 1). Referente al crecimiento relativo, las ciudades latinoamericanas tienen un resultado aún mejor, ya que todas salvo Belo Horizonte tienen un crecimiento de las ventas por arriba del promedio (+59\%). Sobresalen Bogotá con un crecimiento relativo de las ventas de $6162 \%$, lo que la hace la ciudad con el aumento más rápido en el mundo, y Medellín (+907\%). También las empresas en São Paulo, Santiago de Chile, Osasco y Caracas expandieron sus ventas fuertemente, mientras las de la Ciudad de México retroceden, aunque su crecimiento todavía está por arriba del promedio de todas las ciudades. Relativo al desarrollo de las ganancias de las empresas Forbes 2000 con sede en América Latina los resultados son mixtos. La mayoría de las ciudades latinoamericanas tienen un crecimiento fuerte de las ganancias de sus empresas, destacando, en términos absolutos, São Paulo, la Ciudad de México y Bogotá, que se ubican en las Top 50 ciudades. En Monterrey y, sobre todo, en Rio de Janeiro, sin embargo, las ganancias absolutas disminuyeron notablemente. En cuanto al crecimiento relativo, la mayoría de las ciudades latinoamericanas tiene un crecimiento relativo de las ganancias por arriba del promedio de las Forbes 2000 (+ 70\%), distinguiéndose Bogotá como la primera de todas las ciudades de la lista Forbes 2000 (+ 8 654\%). Destacan también Caracas, São Paulo, Osasco y Medellín con un crecimiento relativo muy fuerte, meintras las empresas con sede en la Ciudad de México expandieron sus ganancias por 144 por ciento.

En promedio, el margen de beneficio operativo de las empresas con sede en América Latina excede con $7.7 \%$ ligeramente el promedio de las Forbes Global 2000 (7.5\%). Existen, no obstante, altas diferencias regionales. Mientras las empresas tanto en las dos ciudades brasileras con las empresas más expansivas -São Paulo y Rio de Janeiro- como en Santiago de Chile tienen un rendimiento relativamente bajo, las otras ciudades grandes de América Latina (Caracas, Bogotá, la Ciudad de México, Lima y Brasilia) se colocan muy por arriba del promedio de las Forbes Global 2000. 


\section{Cuadro 1. Ventas y ganancias de las Forbes Global 2000, billones de US-dolares, 2014}

\begin{tabular}{|c|c|c|c|}
\hline \multicolumn{4}{|c|}{2014} \\
\hline & & Ventas (\$bil) & $\%$ del total \\
\hline 1 & Tokio & 3108.70 & 8.12 \\
\hline 2 & Beijing & 2340.90 & 6.12 \\
\hline 3 & Londres & 1489.80 & 3.89 \\
\hline 4 & Paris & 1254.60 & 3.28 \\
\hline 5 & Nueva York & 1114.10 & 2.91 \\
\hline 34 & São Paulo & 217.40 & 0.57 \\
\hline 35 & Rio de Janeiro & 217.30 & 0.57 \\
\hline 65 & Ciudad de México & 127.60 & 0.33 \\
\hline 93 & Santiago & 87.50 & 0.23 \\
\hline 108 & Osasco & 74.10 & 0.19 \\
\hline 116 & Brasilia & 68.60 & 0.18 \\
\hline 123 & Monterrey & 64.50 & 0.17 \\
\hline 141 & Bogotá & 50.70 & 0.13 \\
\hline 243 & Porto Alegre & 22.10 & 0.06 \\
\hline 378 & Medellín & 10.00 & 0.03 \\
\hline 456 & Belo Horizonte & 6.80 & 0.02 \\
\hline 469 & São Jose dos Campos & 6.30 & 0.02 \\
\hline 523 & Lima & 4.50 & 0.01 \\
\hline 536 & Caracas & 4.10 & 0.01 \\
\hline 563 & Jaragua do Sul & 3.20 & 0.01 \\
\hline 566 & Barueri & 3.10 & 0.01 \\
\hline 635 & Maracaibo & 1.60 & 0.00 \\
\hline \multicolumn{4}{|c|}{2014} \\
\hline \multicolumn{4}{|c|}{ Ganancias (\$bil) \% del total } \\
\hline 1 & Beijing & 240.20 & 8.23 \\
\hline 2 & Nueva York & 155.60 & 5.33 \\
\hline 3 & Tokio & 150.30 & 5.15 \\
\hline 4 & Londres & 109.40 & 3.75 \\
\hline 5 & Washington & 86.70 & 2.97 \\
\hline 41 & Ciudad de México & 16.10 & 0.55 \\
\hline 43 & São Paulo & 15.50 & 0.53 \\
\hline 67 & Bogotá & 8.80 & 0.30 \\
\hline 68 & Rio de Janeiro & 8.70 & 0.30 \\
\hline 83 & Brasilia & 7.30 & 0.25 \\
\hline
\end{tabular}




\begin{tabular}{|l|l|l|l|}
\hline 104 & Osasco & 5.60 & 0.19 \\
\hline 144 & Santiago & 3.20 & 0.11 \\
\hline 178 & Monterrey & 2.40 & 0.08 \\
\hline 241 & Belo Horizonte & 1.40 & 0.05 \\
\hline 264 & Barueri & 1.20 & 0.04 \\
\hline 271 & Caracas & 1.20 & 0.04 \\
\hline 299 & Medellín & 1.00 & 0.03 \\
\hline 375 & Porto Alegre & 0.60 & 0.02 \\
\hline 392 & Lima & 0.60 & 0.02 \\
\hline 492 & Jaragua do Sul & 0.40 & 0.01 \\
\hline 507 & Dos Campos São Jose & 0.40 & 0.01 \\
\hline 551 & Maracaibo & 0.30 & 0.01 \\
\hline
\end{tabular}

\begin{tabular}{|c|c|c|c|c|c|c|}
\hline \multicolumn{4}{|c|}{2014} & \multirow[b]{3}{*}{1} & \multirow{2}{*}{\multicolumn{2}{|c|}{ Ventas $2006-14 \%$ crecimiento }} \\
\hline & & \multicolumn{2}{|c|}{ Ganancias (\$bil) \% del total } & & & \\
\hline 1 & Beijing & 240.20 & 8.23 & & Bogotá & 6161.73 \\
\hline 2 & Nueva York & 155.60 & 5.33 & 2 & Shanghai & 1962.87 \\
\hline 3 & Tokio & 150.30 & 5.15 & 3 & Shenzhen & 1254.13 \\
\hline 4 & Londres & 109.40 & 3.75 & 4 & Makati City & 1112.00 \\
\hline 5 & Washington & 86.70 & 2.97 & 5 & Summit & 1100.00 \\
\hline 41 & Cd. de México & 16.10 & 0.55 & 11 & Medellín & 907.07 \\
\hline 43 & São Paulo & 15.50 & 0.53 & 28 & São Paulo & 507.85 \\
\hline 67 & Bogotá & 8.80 & 0.30 & 34 & Santiago & 387.35 \\
\hline 68 & Rio de Janeiro & 8.70 & 0.30 & 40 & Osasco & 326.58 \\
\hline 83 & Brasilia & 7.30 & 0.25 & 48 & Caracas & 301.98 \\
\hline 104 & Osasco & 5.60 & 0.19 & 72 & Brasilia & 201.18 \\
\hline 144 & Santiago & 3.20 & 0.11 & 75 & Porto Alegre & 198.78 \\
\hline 178 & Monterrey & 2.40 & 0.08 & 89 & Rio de Janeiro & 164.13 \\
\hline 241 & Belo Horizonte & 1.40 & 0.05 & 105 & Lima & 137.70 \\
\hline 264 & Barueri & 1.20 & 0.04 & 183 & Ciudad de México & 77.76 \\
\hline 271 & Caracas & 1.20 & 0.04 & 205 & Monterrey & 66.46 \\
\hline 299 & Medellín & 1.00 & 0.03 & 209 & $\begin{array}{l}\text { Dos Campos São } \\
\text { Jose }\end{array}$ & 64.16 \\
\hline 375 & Porto Alegre & 0.60 & 0.02 & 436 & Belo Horizonte & -7.13 \\
\hline 392 & Lima & 0.60 & 0.02 & & & \\
\hline 492 & Jaragua do Sul & 0.40 & 0.01 & & & \\
\hline 507 & $\begin{array}{l}\text { Dos Campos } \\
\text { São Jose }\end{array}$ & 0.40 & 0.01 & & & \\
\hline 551 & Maracaibo & 0.30 & 0.01 & & & \\
\hline
\end{tabular}




\begin{tabular}{|c|c|c|c|c|c|}
\hline & \multicolumn{2}{|c|}{ Ganancias 2006-14 absolute } & & \multicolumn{2}{|c|}{ Ganancias $2006-14 \%$ crecimiento } \\
\hline 1 & Beijing & 207.50 & 1 & Bogotá & 8654.30 \\
\hline 2 & Tokio & 77.52 & 2 & Casablanca & 4230.50 \\
\hline 3 & Washington & 77.42 & 3 & Bezons & 3380.00 \\
\hline 4 & Moscú & 62.11 & 4 & $\begin{array}{l}\text { Metropolitan City } \\
\text { Ulsan }\end{array}$ & 3349.20 \\
\hline 5 & McLean & 50.03 & 5 & Pembroke & 3348.25 \\
\hline 24 & São Paulo & 12.65 & 27 & Caracas & 531.58 \\
\hline 34 & $\begin{array}{l}\text { Ciudad de } \\
\text { México }\end{array}$ & 9.52 & 35 & São Paulo & 450.19 \\
\hline 38 & Bogotá & 8.65 & 43 & Osasco & 383.48 \\
\hline 56 & Brasilia & 5.43 & 49 & Medellín & 340.14 \\
\hline 68 & Osasco & 4.41 & 54 & Brasilia & 290.37 \\
\hline 125 & Santiago & 1.74 & 115 & Ciudad de México & 143.87 \\
\hline 162 & Barueri & 1.24 & 140 & Santiago & 115.77 \\
\hline 186 & Caracas & 1.01 & 202 & Lima & 71.82 \\
\hline 227 & Medellín & 0.75 & 311 & Porto Alegre & 11.20 \\
\hline 337 & Jaragua do Sul & 0.39 & 354 & Belo Horizonte & -13.25 \\
\hline 392 & Maracaibo & 0.28 & 375 & $\begin{array}{l}\text { Dos Campos São } \\
\text { Jose }\end{array}$ & -23.38 \\
\hline 416 & Lima & 0.24 & 390 & Rio de Janeiro & -34.50 \\
\hline 496 & Porto Alegre & 0.06 & 397 & Monterrey & -41.88 \\
\hline 562 & $\begin{array}{l}\text { Rio Grande, Rio } \\
\text { Grande do Sul }\end{array}$ & -0.07 & & & \\
\hline 581 & $\begin{array}{l}\text { São Jose dos } \\
\text { Campos }\end{array}$ & -0.11 & & & \\
\hline 622 & Belo Horizonte & -0.22 & & & \\
\hline 809 & Monterrey & -1.72 & & & \\
\hline 838 & Rio de Janeiro & -4.61 & & & \\
\hline
\end{tabular}

Fuente: elaboración propia, basada en Forbes 2014.

El segundo indicador que se analiza aquí es el valor agregado bruto en servicios al productor. Estos servicios son, como se argumentó, indispensables para las operaciones, tanto de empresas globales como para los mercados financieros. Los resultados del análisis parecen, por un lado, al patrón geográfico de las sedes de las empresas más grandes. En 2013, las ciudades con más valor agregado en los servicios al productor son Nueva York (con 9.1\% del total de las 126 ciudades), seguida por Tokio, Paris, Londres y Los Ángeles (ver cuadro 2). Por el otro, se observan también diferencias importantes, especialmente con referencia a las 
ciudades chinas: Beijing solo viene en el 15 lugar, con una participación de 1.7\% en el valor agregado bruto en servicios al productor.

En cuanto a las ciudades latinoamericanas también se muestran diferencias, ya que generan en total $5.6 \%$ del valor agregado bruto en servicios al productor -más que el doble que la participación en las ventas y ganancias de las empresas más grandes-. Destacan São Paulo y la Ciudad de México con 1.2\% cada una (lugares 21 y 22, junto a Nagoya y Phoenix), pero también Santiago de Chile y Buenos Aires -ausente en la lista de Forbes- se encuentran entre las 50 ciudades con más participación en la producción de los servicios altos. Por el otro, Rio de Janeiro, que en el listado de las empresas más grandes es una de las ciudades latinoamericanas altamente clasificada, tiene una producción relativamente baja en este sector.

Cuadro 2: Valor agregado bruto en servicios financieros, inmobiliarios e actividades de negocio, 2013, millones de US-\$ corrientes

\begin{tabular}{|c|c|c|c|c|c|c|}
\hline \multicolumn{4}{|c|}{$\begin{array}{l}\text { Valor Agregado Bruto } 2013 \text { millones de US-\$ corrientes } \\
\qquad \% \text { del total }\end{array}$} & \multicolumn{3}{|c|}{ 2006-13 cambio absoluto } \\
\hline 1 & Nueva York & 617850.2 & 9.08 & 1 & Paris & 107943.4 \\
\hline 2 & Tokio & 412636.9 & 6.07 & 2 & Nueva York & 105164.4 \\
\hline 3 & Paris & 357247.8 & 5.25 & 3 & Beijing & 82123.0 \\
\hline 4 & Londres & 324595.4 & 4.77 & 4 & Moscú & 77088.8 \\
\hline 5 & Los Ángeles & 296933.0 & 4.37 & 5 & Shanghai & 67170.8 \\
\hline 21 & São Paulo & 82364.8 & 1.21 & 12 & São Paulo & 40975.9 \\
\hline 22 & $\begin{array}{l}\text { Ciudad de } \\
\text { México }\end{array}$ & 80574.0 & 1.18 & 20 & Santiago & 27883.4 \\
\hline 38 & Santiago & 52292.7 & 0.77 & 25 & Buenos Aires & 22837.5 \\
\hline 43 & Buenos Aires & 42048.6 & 0.62 & 36 & Bogotá & 17160.8 \\
\hline 57 & Bogotá & 30392.7 & 0.45 & 41 & $\begin{array}{l}\text { Ciudad de } \\
\text { México }\end{array}$ & 15116.9 \\
\hline 68 & Rio de Janeiro & 24281.6 & 0.36 & 56 & Rio de Janeiro & 10039.6 \\
\hline 80 & Lima & 16682.3 & 0.25 & 60 & Lima & 9402.2 \\
\hline 92 & Caracas & 9379.9 & 0.14 & 78 & Caracas & 5099.5 \\
\hline 96 & Montevideo & 8122.3 & 0.12 & 81 & Montevideo & 4842.3 \\
\hline 100 & Santo Domingo & 6097.6 & 0.09 & 88 & Santo Domingo & 3319.5 \\
\hline 101 & Quito & 6037.9 & 0.09 & 89 & San José & 3291.2 \\
\hline 105 & San José & 5301.3 & 0.08 & 91 & Quito & 2876.4 \\
\hline 108 & Guayaquil & 5055.6 & 0.07 & 95 & Guayaquil & 2431 \\
\hline 110 & Salvador & 4661.9 & 0.07 & 96 & Salvador & 2281.9 \\
\hline 115 & Guatemala City & 4025.8 & 0.06 & 109 & Guatemala & 1451.4 \\
\hline 126 & Santa Cruz & 571.3 & 0.01 & 123 & Santa Cruz & 344.9 \\
\hline
\end{tabular}




\begin{tabular}{|c|c|c|c|c|}
\hline \multicolumn{2}{|c|}{ 2006-13 cambio relativo (\%) } & \multicolumn{3}{|c|}{ Valor Agregado Bruto US-\$ / capita, 2013} \\
\hline Tianjin & 535.7 & 1 & Bruselas & 351695.4 \\
\hline Wuhan & 395.5 & 2 & Oslo & 344796.7 \\
\hline Shenzhen & 327.6 & 3 & Ginebra & 326587.5 \\
\hline Guangzhou & 326.5 & 4 & Paris & 314973.3 \\
\hline Ho Chi Minh City & 294.6 & 5 & Doha & 307117.4 \\
\hline San José & 163.7 & 53 & Santiago & 135627.9 \\
\hline Santa Cruz & 152.3 & 65 & Santo Domingo & 98118.9 \\
\hline Montevideo & 147.6 & 70 & Ciudad de México & 79998.5 \\
\hline Bogotá & 129.7 & 82 & Montevideo & 63934.2 \\
\hline Lima & 129.1 & 92 & Buenos Aires & 52014.8 \\
\hline Santo Domingo & 119.5 & 95 & São Paulo & 50443.4 \\
\hline Caracas & 119.1 & 96 & Caracas & 50406.0 \\
\hline Buenos Aires & 118.9 & 104 & Quito & 43754.9 \\
\hline Santiago & 114.2 & 107 & Guayaquil & 38111.0 \\
\hline São Paulo & 99.0 & 108 & Bogotá & 38067.3 \\
\hline Salvador & 95.9 & 109 & San José & 37663.0 \\
\hline Guayaquil & 92.6 & 111 & Lima & 36805.6 \\
\hline Quito & 91.0 & 114 & Rio de Janeiro & 27812.8 \\
\hline Rio de Janeiro & 70.5 & 120 & Salvador & 15171.2 \\
\hline Guatemala City & 56.4 & 122 & Santa Cruz & 9933.8 \\
\hline Ciudad de México & 23.1 & & & \\
\hline
\end{tabular}

Fuente: elaboración propia, basada en Euromonitor 2014.

En cuanto al impacto de la crisis en el sector de los servicios al productor en América Latina, se confirman grosso modo los resultados del análisis de la lista de Forbes: la recesión global impactó menos en esta región, lo que resulta en un crecimiento por arriba del promedio en casi todas las ciudades (2006-2013). Mientras en el total de las 126 ciudades analizadas el valor agregado bruto en los servicios al productor creció 38\%, en América Latina fue $81 \%$. En términos absolutos, destaca el crecimiento en São Paulo, Santiago de Chile y Buenos Aires, mientras referente al crecimiento relativo de los servicios al productor son las ciudades con un sector todavía relativamente pequeño que sobresalen: en San José, Santa Cruz, Montevideo, Bogotá, Lima, Santo Domingo y Caracas, la producción se duplicó. En las dos ciudades brasileras más importantes el crecimiento no fue tan pronunciado, pero todavía por arriba del promedio, mientras la Ciudad de México fue más afectada por la crisis: Aquí el valor agregado bruto en servicios al productor creció por solamente 23 por ciento. 
En cuanto a la productividad del sector de los servicios al productor (valor agregado por persona ocupada), el balance para las ciudades latinoamericanas es mucho menos favorable. Todas salvo Santiago de Chile tienen una producción per cápita por debajo del promedio de las 126 ciudades, y eso en parte es significativo: en la Ciudad de México -después de Santiago y Santo Domingo la tercera en América Latina- el output por persona queda 36\% por debajo del promedio, en Buenos Aires 59\%, en São Paulo 60\% y en Rio de Janeiro 78\%. Dicho de otra manera, una persona ocupada en el sector de los servicios al productor en la Ciudad de México agrega por hora de trabajo unos 150 US-\$ menos de valor que su colega en Bruselas, las ciudad con la productividad más alta. ${ }^{6}$

El tercer indicador para evaluar la centralidad económica es la 'Global Network Connectivity' (GNC), que estima las conexiones entre ciudades globales (Taylor et al. 2011). En 2013, las ciudades más conectadas son Londres, Nueva York (con 94\% de la conectividad de Londres), Hong-Kong (78\%), Paris (72\%) y Singapur (68\%) (cuadro 3). Llama la atención la integración fuerte de ciudades asiáticas -seis de las diez ciudades más conectadas se encuentran en Asia (además de Hong Kong y Singapur son Tokio, Shanghái, Dubái y Beijing)-. También llama la atención que algunas ciudades latinoamericanas tienen una GNC muy alta: São Paulo se coloca en el lugar 16, con 58\% de la conectividad de Londres, justo a Frankfurt, mientras la Ciudad de México viene en el lugar 20 (54\%), detrás de Madrid. Además de São Paulo y la Ciudad de México, GaWC (2014) clasifica también a Buenos Aires y Santiago de Chile como "Alpha World Cities" ("Very important world cities that link major economic regions and states into the world economy"), mientras Lima, Bogotá, Caracas, Montevideo, Rio de Janeiro, Guatemala, Panamá, San José, y Quito están contadas como "Beta World Cities" ("important world cities that are instrumental in linking their region or state into the world economy"). En la última década (2000-2013), tres de las cuatro "Alpha World Cities" de América Latina -Santiago de Chile, Ciudad de México y São Paulo- aumentaron su conectividad por arriba del promedio de las Top 50 ciudades (55\%), mientras Buenos Aires' integración en la red de ciudades globales se ha disminuido. Los crecimientos en la GNC más fuertes han tenido, sin embargo, ciudades hasta ahora menos conectadas como Querétaro y Puebla, Cali, San Salvador, Cordoba, Guatemala o Lima.

6 Calculando hipotéticamente con 46 semanas de trabajo al año y 40 horas la semana. 
Cuadro 3: 'Global Network Connectivity' de las ciudades globales, 2013

Global Network Connectivity 2013, relativo a Londres

\begin{tabular}{|c|c|c|}
\hline 1 & London & 1.00 \\
\hline 2 & New York & 0.94 \\
\hline 3 & Hongkong & 0.78 \\
\hline 4 & Paris & 0.72 \\
\hline 5 & Singapore & 0.68 \\
\hline 16 & São Paulo & 0.58 \\
\hline 20 & Ciudad de México & 0.54 \\
\hline 29 & Buenos Aires & 0.49 \\
\hline \multirow[t]{10}{*}{44} & Santiago & 0.43 \\
\hline & Lima & 0.35 \\
\hline & Bogotá & 0.34 \\
\hline & Caracas & 0.32 \\
\hline & Montevideo & 0.32 \\
\hline & Rio de Janeiro & 0.29 \\
\hline & Guatemala & 0.28 \\
\hline & Panama & 0.28 \\
\hline & San José & 0.28 \\
\hline & Quito & 0.25 \\
\hline
\end{tabular}

Global Network Connectivity, crecimiento absoluto 2000-2013

\begin{tabular}{|l|l|l|l|}
\hline 1 & Dubai & 0.30 \\
\hline 3 & Shanghai & 0.20 \\
\hline 5 & Beijing & 0.19 \\
\hline & Doha & Moscú & 0.18 \\
\hline & & 0.18 \\
\hline & Querétaro & \\
\hline & Puebla & 0.15 \\
\hline & León & 0.14 \\
\hline & Cali & 0.13 \\
\hline & San Salvador & 0.12 \\
\hline & Cordoba & 0.12 \\
\hline & Santa Cruz & 0.12 \\
\hline & Campinas & 0.12 \\
\hline & San José & 0.10 \\
\hline & Guatemala & 0.10 \\
\hline & Lima & 0.10 \\
\hline
\end{tabular}




\begin{tabular}{|l|l|l|}
\hline & Santiago & \\
\hline & & \\
\hline & Ciudad de México & \\
\hline & & 0.08 \\
\hline & São Paulo & \\
\hline & Montevideo & 0.04 \\
\hline & Quito & 0.04 \\
\hline & & 0.02 \\
\hline & Buenos Aires & 0.02 \\
\hline & Panama & 0.01 \\
\hline & & \\
\hline & Rio de Janeiro & 0.00 \\
\hline
\end{tabular}

Fuente: elaboración propia, basada en GaWC.

\section{Discusión}

El análisis empírico de los patrones geográficos de las actividades económicas centrales arroja varios resultados. En términos generales (que aquí no fue el enfoque principal), sobresale la importancia excepcional de Nueva York y Londres, pero también de Tokio y Paris. Sin duda, son los centros de la economía mundial, y eso respecto al poder económico formal, al poder organizativo y al poder globalizador. Destaca también, sin embargo, que ciudades asiáticas (y, en particular, chinas) están alcanzando, sobre todo con Beijing, Hong-Kong, Singapur y Shanghái mayor centralidad en varios aspectos.

Referente a América Latina, los resultados muestran, primero, que en cuanto al poder económico formal, sus ciudades son marginales, aunque lograron mejorar su posición en el curso de la reciente crisis de la economía mundial. Sobresale São Paulo tanto en cuanto al crecimiento de las ventas como de las ganancias, mientras las empresas de la Ciudad de México y de Bogotá destacan sobre todo por su incremento en las ganancias. Segundo, el análisis revela también que las ciudades latinoamericanas ocupan lugares más centrales en la economía mundial en cuanto al poder organizativo y globalizador. Tanto São Paulo como la Ciudad de México tienen un sector de servicios al productor importante y fuertemente integrado en la red de ciudades globales. Además, en varias ciudades latinoamericanas, tanto 
la producción del sector de los servicios altos como su conectividad se han incrementados notablemente en la última década.

¿Cómo se pueden interpretar estos resultados divergentes? Para comprender la diferencia entre el poder económico formal bastante limitado y el poder organizativo y globalizador más significante hay que subrayar, primero, la falta de vigor del sector manufacturero -tanto privado como estatal- en América Latina. Son los servicios altos y, en particular, el sector financiero que contribuyen la mayoría a las ventas y ganancias de las empresas latinoamericanas listadas en Forbes Global 2000 - 30 a $41 \%$, respectivamente. Tan sólo tres bancos brasileros (Banco Bradesco, Banco do Brasil, Itau Unibanco Holding) contribuyen más que un quinto a las ventas y más que un cuarto a las ganancias de las Forbes Global 2000 de América Latina. El segundo sector más importante es la minería contribuyendo $20 \%$ a las ventas y $27 \%$ a las ganancias. En este sector, destacan empresas semiestatales como Petrobas y Ecopetrol que beneficiaron del auge del precio de petroleo desde $2001 .^{7}$ En tercer lugar viene el sector de telecommunicación, contribuyendo 8 y 10\% a las ventas y ganancias. La empresa más importante en este sector es América Móvil, que en México goza de un cuasi-monopolio, heredido de su anterior empresa matriz Telmex.

Segundo, los datos analizados muestran que São Paulo y la Ciudad de México, y en menor grado también Santiago de Chile, Buenos Aires, Bogotá, Rio de Janeiro y Caracas, son nodos importantes para la organización y la gestión de cadenas productivas globales, ya que estas ciudades contienen clusteres importantes de servicios al productor. Sin embargo, es cuestionable denotarles a estas empresas "latinoamericanas" en términos convencionales, que suelen equiparar la nacionalidad legal de una empresa con una "economía nacional" y hasta con un "interés nacional". Sin embargo, para una discusión de la posición de América Latina en la centralidad de la economía mundial es clave comprender que los actores del poder organizativo y globalizador de sus ciudades -o sea, las empresas de servicios al productor responsables para la "nodalizaciòn" de las ciudades latinoamericanas- en muchos casos son oficinas locales de empresas globales. No obstante las diferencias regionales y sectorales que puedan existir (p. e. entre el sector financiero en Brasil y en México), en general el sector de servicios al productor esta altamente -y cada vez más- globalizado (Daniels, 2007). ${ }^{8}$ Aunque

\footnotetext{
7 Hay que recordar en este contexto que la empresa petrolera mexicana Pemex, según Fortune 500 la segunda empresa más grande de América Latina -detrás de Petrobas- no es empresa pública por lo cual no esta listada por Forbes.

8 Tomemos el caso de la Ciudad de México: De los diez bancos más grandes en el país, seis pertenecen a instituciones financieras transnacionales. Controlan $69 \%$ de los activos de todos los bancos en México (http://rankings.americaeconomia.com/2012/bancos/ranking250.php), - cuatro veces más que los cuatro en propiedad del capital privado nacional (Banorte, Inbursa, Interacciones, Banco del Bajío). También en servicios legales, de contabilidad o de asesoría, la presencia de firmas internacionales salta a la vista: 93 de las 175 empresas de servicios al productor globales más importantes analizados por GaWC están representados con oficinas en la Ciudad de México. Aunque no se sabe exactamente qué la contribución de estas firmas al valor agregado del sector de los servicios al productor en la Ciudad de México será, tanto el tamaño de las oficinas como la cantidad de servicios que prestan a clientes
} 
sí es cierto que las empresas de servicios al productor legalmente son mexicanas, brasileras, etc., ${ }^{9}$ asignarlas una identidad nacional es conceptualmente dificil de sostener, ya que su recurso central -conocimientos- se produce relacionalmente, en redes globales (Bathelt/Glückler, 2011). Es, por ende, analíticamente más preciso llamarles transnacionales, ya que estas empresas se caracterizan por estrategias organizativas trans-fronterizas y decentrales. Compartiendo "knowledge, resources and responsibilities" (UNCTAD, 2014) entre las unidades de la empresa, una transnacional es:

a complex configuration of assets and capabilities that are distributed, yet specialized. Furthermore, the company integrates the dispersed resources through strong interdependencies... Such interdependencies may be reciprocal rather than sequential... they are specifically designed to build self-enforcing cooperation among interdependent units. (Bartlett and Ghoshal, 2002:69).

Dado esta fuerte interdependencia entre las unidades de las empresas de los servicios al productor, se puede concluir como hipótesis de trabajo para futuras investigaciones, que la centralidad que algunas ciudades latinoamericanas tienen en la economía mundial resulta de sus relaciones exógenas más que de su potencia endógena. São Paulo y la Ciudad de México, y en menor grado también Santiago de Chile, Buenos Aires, Bogotá, Rio de Janeiro y Caracas funcionan como bisagras entre actividades económicas en Brasil, México, etc., y como tal forman parte de una centralidad económica que geográficamente esta dispersa, estructurada en la red de ciudades globales.

importantes hacen a suponer que es buena parte (para un estudio de caso de la Ciudad de México ver Parnreiter, 2010).

9 En el sector de los servicios al productor hay dos maneras de organización: Mientras en muchas ramas las empresas globales operan a través de alianzas y redes globales, con los socios formando empresas formalmente independientes ( $p$. e. en el caso de las empresas legales o de contabilidad), en otras ramas (p. e. servicios financieros) se trata más de filiales directas de empresas extranjeras. 


\section{Bibliografía}

Bartlett, Christopher A./Sumantra Ghoshal: 2002 Managing across borders: the transnational solution. Boston,

Mass.: Harvard Business School Press.

Bathelt,H.; Glückner,J. (2011): The Relational Economy. Geographies of Knowing and Learning. Oxford

Brown, E., B. Derudder, C. Parnreiter, W. Pelupessy, P.J. Taylor \& F. Witlox (2010), World City Networks and Global Commodity Chains: Towards a World Systems Integration. Global Networks 10, 12-34.

Bryson, John R./Peter W. Daniels: 2007 Worlds of services: from local service economies to offshoring or global sourcing. In: Bryson, John R./Peter W. Daniels (eds.): The Handbook of Service Industries. Edward Elgar, Cheltenham, 1-18.

Coe, Neil M./ Dicken, Peter/ Hess, Martin: 2008 Global production networks: Realizing the potential. In: Journal of Economic Geography, 8, 3, 271-295.

Daniels, Peter W. : 2007 A global service economy? In: Bryson, John R./Peter W. Daniels (eds.): The Handbook of Service Industries. Edward Elgar, Cheltenham, 103-125.

Dicken, Peter: 2011 Global Shift: Mapping the Changing Contours of the World Economy. Sage Publications. London.

Dunning, John H./Sarianna Lundan: 2008 Multinational Enterprises and the Global Economy. Cheltenham: Edward Elgar, Second Edition.

Euromonitor International: Passport Cities.

GaWC 2014: The World According to GaWC. http://www.lboro.ac.uk/gawc/gawcworlds.html Gereffi, Gary/Miguel Korzeniewicz (eds.): 1994 Commodity Chains and Global Capitalism. Westport: Praeger. Harvey, David: 1985 The Urbanization of Capital. Blackwell. Oxford.

Ferreira, Francisco H. G. Julian Messina, Jamele Rigolini, Luis-Felipe López-Calva, Maria Ana Lugo and Renos

Vakis: Economic Mobility and the Rise of the Latin American Middle Class. The World Bank 2013.

The World's Biggest Public Companies. http://www.forbes.com/global2000/list/

Hopkins, Terence K./Immanuel Wallerstein: 1977 Patterns of Development of the Modern World System.

Research Proposal. In: Review, Bd. 1, Nr. 2, 111-145.

International Monetary Fund (IMF) 2014a: World Economic Outlook Database. http://www.IMF.org/external/pubs/ $\mathrm{ft} /$ weo/2014/02/weodata/index.aspx

International Monetary Fund (IMF) 2014b: World Economic and Financial Surveys. Regional Economic Outlook. Western Hemisphere. Rising Challenges.

Jacobs, Jane: 1997 Still Challenging the Way We Think About Cities. Los Angeles Times, October 12, 1997. http://articles.latimes.com/print/1997/oct/12/opinion/op-41978

MGI: 2012 Urban world: Cities and the rise of the consuming class. McKinsey Global Institute

OCDE 2013: Perspectivas económicas de América Latina 2014 Perspectivas económicas de América Latina

2014. Logística y competitividad para el desarrollo.

Parnreiter, Christof: 2010 Global cities in Global Commodity Chains: exploring the role of Mexico City in the geography of global economic governance. In: Global Networks 10, 1, 35-53.

Sassen, Saskia: 1991, The Global City. New York, London, Tokyo. Princeton University Press. Princeton.

Scott, Allen J./Michael Storper: 2014 The Nature of Cities: The Scope and Limits of Urban Theory. In: International Journal of Urban and Regional Research, early view DOI: 10.1111/1468-2427.12134

Schumpeter, Joseph (1987 [1934]): Theorie der wirtschaftlichen Entwicklung. Berlin.

Smith, David A.: 2000 Urbanization in the World-System: A Retrospective and Prospective. In: Hall, Thomas D. (ed): A World-Systems Reader. New Perspectives on Gender, Urbanism, Cultures, Indigenous Peoples, 
and Ecology. Rowman \& Littlefield Publishers. Lanham, 143-168.

Taylor, Peter: 2004 World City Network. A global urban analysis. Routledge. London. Taylor, P.J., P. Ni, B.

Derudder, M. Hoyler, J. Huang \& F. Witlox, eds. (2011), Global Urban Analysis. A Survey of Cities in

Globalization. London: Earthscan

United Nations Conference on Trade and Development (UNCTAD): 2004 World Investment Report 2004. The Shift Towards Services. United Nations. New York.

United Nations Conference on Trade and Development (UNCTAD): (2013): World investment report 2013 Global

Value Chains: Investment and Trade for Development. United Nations. New York.

United Nations Conference on Trade and Development (UNCTAD): 2014 Transnational corporations statistics, http://unctad.org/en/Pages/DIAE/Transnational-Corporations-Statistics.aspx

Wallerstein, Immanuel: 1983 Historical Capitalism. Verso. London.

Wallerstein, Immanuel: 2001 Unthinking Social Science. Temple University Press. Philadelphia.

World Bank: 2013 Planning, Connecting, and Financing Cities-Now. Priorities for City Leaders. World Bank. 